Correction

\title{
Correction: Liquori et al. Acute Promyelocytic Leukemia: A Constellation of Molecular Events around a Single PML-RARA Fusion Gene. Cancers 2020, 12, 624
}

\author{
Alessandro Liquori ${ }^{1,+}+(\mathbb{D})$, Mariam Ibañez ${ }^{2,3,+}$, Claudia Sargas ${ }^{1}$, Miguel Ángel Sanz ${ }^{2,3}$ (D) Eva Barragán ${ }^{2,3}$ (D) \\ and José Cervera $2,3, *$ (D) \\ 1 Accredited Research Group in Hematology and Hemotherapy, Instituto de Investigación Sanitaria La Fe, \\ 46026 Valencia, Spain; alessandro_liquori@iislafe.es (A.L.); claudia_sargas@iislafe.es (C.S.) \\ 2 Department of Hematology, Hospital Universitario y Politécnico La Fe, 46026 Valencia, Spain; \\ ibanyez_marcom@gva.es (M.I.); Miguel.Sanz@uv.es (M.Á.S.); barragan_eva@gva.es (E.B.) \\ 3 Centro de Investigación Biomédica en Red de Cáncer (CIBERONC), 28029 Madrid, Spain \\ * Correspondence: cervera_jos@gva.es \\ + A.L. and M.I. contributed equally to this work.
}

Citation: Liquori, A.; Ibañez, M.; Sargas, C.; Sanz, M.Á.; Barragán, E.; Cervera, J. Correction: Liquori et al. Acute Promyelocytic Leukemia: A Constellation of Molecular Events around a Single PML-RARA Fusion Gene. Cancers 2020, 12, 624. Cancers 2021, 13, 3440. https://doi.org/ $10.3390 /$ cancers 13143440

Received: 12 May 2021

Accepted: 29 June 2021

Published: 9 July 2021

Publisher's Note: MDPI stays neutral with regard to jurisdictional claims in published maps and institutional affiliations.

The authors wish to make the following corrections to this paper [1]:

In the last row of Table 2, translocation is $\mathrm{t}(3 ; 17)(\mathrm{q} 26 ; \mathrm{q} 21)$ instead of $\mathrm{t}(1 ; 17)(\mathrm{q} 42 ; \mathrm{q} 21)$.

In the last row of Table 2, ATO sensitivity should be changed to ND (not determined) instead of "Sensitive".

The new Table 2 is shown below: 
Table 2. APL molecular variants.

\begin{tabular}{|c|c|c|c|c|c|c|c|c|c|c|}
\hline $\begin{array}{l}\text { APL Molecular } \\
\text { Variants }\end{array}$ & Translocations & $\begin{array}{c}\text { ATRA } \\
\text { Sensitivity }\end{array}$ & $\begin{array}{c}\text { ATO } \\
\text { Sensitivity }\end{array}$ & $\begin{array}{c}\text { No. of } \\
\text { Cases } \\
\text { Reported }\end{array}$ & $\begin{array}{l}\text { Gene Other } \\
\text { Than } P M L, \\
\text { Breakpoint }\end{array}$ & $\begin{array}{l}\text { Gene Other } \\
\text { Than } P M L, \\
\text { Splice Site }\end{array}$ & Genomic Insertions & $\begin{array}{c}\text { RARA, } \\
\text { Breakpoint }\end{array}$ & $\begin{array}{c}\text { RARA, } \\
\text { Splice Site }\end{array}$ & Reference \\
\hline \multirow{2}{*}{$\begin{array}{c}\text { ZBTB16 } \\
(P L Z F)-R A R A\end{array}$} & \multirow{2}{*}{$\mathrm{t}(11 ; 17)(\mathrm{q} 23 ; \mathrm{q} 21)$} & \multirow{2}{*}{$\begin{array}{l}\text { Poorly } \\
\text { responsive }\end{array}$} & \multirow{2}{*}{$\begin{array}{c}\text { Poorly } \\
\text { responsive }\end{array}$} & \multirow{2}{*}{$>30[85]$} & Intron 3 & CAGgtaggc & \multirow{19}{*}{$\begin{array}{c}79 \text { nt with no homology } \\
\text { to sequences in the } \\
\text { GenBank or EMBL } \\
\text { databases }\end{array}$} & Intron 2 & ctctagCCA & [102] \\
\hline & & & & & Intron 5 & CAGgtagag & & Intron 2 & ctctagCCA & [103] \\
\hline \multirow[t]{2}{*}{ NPM1-RARA } & \multirow[t]{2}{*}{$\mathrm{t}(5 ; 17)(\mathrm{q} 35 ; \mathrm{q} 21)$} & \multirow[t]{2}{*}{ Sensitive } & \multirow[t]{2}{*}{ ND } & \multirow[t]{2}{*}{$5[103]$} & Intron 5 & CAGgtagag & & Intron 2 & ctctagCCA & [104] \\
\hline & & & & & Intron 4 & TAGgtatgt & & Intron 2 & ctctagCCA & [103] \\
\hline $\begin{array}{c}\text { NUMA1 } \\
\text { (NUMA)-RARA }\end{array}$ & $\mathrm{t}(11 ; 17)(\mathrm{q} 13 ; \mathrm{q} 21)$ & Sensitive & ND & 1 & Intron 23 & CAGgtgagg & & Intron 2 & ctctagCCA & [89] \\
\hline STAT5B-RARA & $\operatorname{der}(17)$ & $\begin{array}{l}\text { Poorly } \\
\text { responsive }\end{array}$ & $\begin{array}{c}\text { Poorly } \\
\text { responsive }\end{array}$ & 11 [105] & Intron 15 & CTCgtgagt & & Intron 2 & ctctagCCA & {$[90,105]$} \\
\hline $\begin{array}{l}\text { PRKAR1A- } \\
\quad \text { RARA }\end{array}$ & $\begin{array}{c}\mathrm{t}(17 ; 17)(\mathrm{q} 21 ; \mathrm{q} 24) \\
\text { or } \\
\operatorname{del}(17)(\mathrm{q} 21 ; \mathrm{q} 24)\end{array}$ & Sensitive & Sensitive & 1 & Intron 2 & AAGgtaaaa & & Intron 2 & ctctagCCA & [91] \\
\hline FIP1I1-RARA & $t(4: 17)(a 12: q 21)$ & Sensitive in 1 & ND & 2 & Intron 15 & ATGgtaagt & & Intron 2 & ctctagCCA & [92] \\
\hline$B C O R-R A R A$ & $\mathrm{t}(\mathrm{X}: 17)(\mathrm{p} 11: \mathrm{q} 21)$ & Sensitive in 2 & Insensitive & & Intron 12 & CAGgtatga & & Intron 2 & ctctagCCA & [93] \\
\hline BCOR-RARA & $\mathrm{t}(\mathrm{X} ; 17)(\mathrm{p} 11 ; \mathrm{q} 21)$ & cases & in 1 case & 2 & Exon 12 & CAGgtagaa & & Intron 2 & ctctagCCA & [107] \\
\hline $\begin{array}{l}\text { NABP1 } \\
(O B F C 2 A)- \\
\text { RARA }\end{array}$ & $\mathrm{t}(2 ; 17)(\mathrm{q} 32 ; \mathrm{q} 21)$ & $\begin{array}{l}\text { Sensitive } \\
\text { in vitro }\end{array}$ & ND & 1 & Intron 5 & TGGgtaaga & & Intron 2 & ctctagCCA & [94] \\
\hline TBL1XR1 & $t(3 \cdot 17)(a 26 \cdot a 21)$ & & ND & $4[108]$ & Intron 5 & CAAgtgagc & & Intron 2 & ctctagCCA & [95] \\
\hline (TBLR1)-RARA & $\mathrm{t}(3 ; 1)$ & $\ln$ & ND & $4[108]$ & Intron 5 & CAAgtgagc & & Intron 2 & ctctagCCA & [108] \\
\hline GTF2I-RARA & $\mathrm{t}(7 ; 17)(\mathrm{q} 11 ; \mathrm{q} 21)$ & Sensitive & Sensitive & 1 & Intron 6 & TAGgtaagt & & Intron 2 & ctctagCCA & [96] \\
\hline \multirow{4}{*}{ IRF2BP2-RARA } & \multirow{4}{*}{$\mathrm{t}(1 ; 17)(\mathrm{q} 42 ; \mathrm{q} 21)$} & \multirow{4}{*}{ Sensitive } & \multirow{4}{*}{ Sensitive } & \multirow{4}{*}{5 [109] } & Exon 2 & TGTcccetg & & Intron 2 & ctctagCCA & {$[97,109]$} \\
\hline & & & & & Exon 1 & AAGgtgcgg & & Intron 2 & ctctagCCA & [110] \\
\hline & & & & & Intron 1 & CAGgtaggg & & Intron 2 & ctctagCCA & [111] \\
\hline & & & & & Exon 1 & CAGgcaggt & & Intron 2 & ctctagCCA & {$[111,112]$} \\
\hline FNDC3B-RARA & $\mathrm{t}(3 ; 17)(\mathrm{q} 26 ; \mathrm{q} 21)$ & Sensitive & ND & 1 & Intron 24 & AAGgtgtgt & & Intron 2 & ctctagCCA & [98] \\
\hline
\end{tabular}

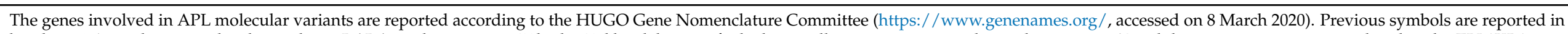

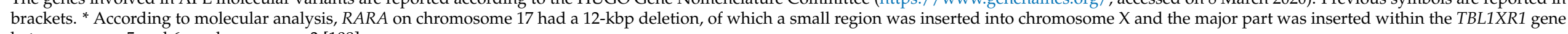
between exons 5 and 6 on chromosome 3 [108]. 
The authors would like to apologize for any inconvenience caused to the readers by these changes. The original manuscript has been updated.

Funding: This research received no external funding.

Conflicts of Interest: The authors declare no conflict of interest.

\section{Reference}

1. Liquori, A.; Ibañez, M.; Sargas, C.; Sanz, M.Á.; Barragán, E.; Cervera, J. Acute Promyelocytic Leukemia: A Constellation of Molecular Events around a Single PML-RARA Fusion Gene. Cancers 2020, 12, 624. [CrossRef] [PubMed] 